\title{
Axillary Ultrasound Before Neoadjuvant Chemotherapy for Breast Cancer: Don't Discount the Benefits Yet!
}

\author{
Theresa L. Schwartz, MD, FACS ${ }^{1}$ and Julie A. Margenthaler, MD, FACS ${ }^{2}$ \\ ${ }^{1}$ Department of Surgery, St. Louis University Health Sciences Center, St. Louis, MO; ${ }^{2}$ Department of Surgery, Washington \\ University School of Medicine, St. Louis, MO
}

Axillary lymph node status remains a top prognostic indicator for patients with breast cancer. It has been well established that the extent of nodal involvement plays a key role in the risk for both local recurrence and overall survival, and nodal staging has a tremendous impact on systemic therapy and radiotherapy treatment recommendations.

During the last 25 years, significant changes in our nodal assessment techniques have occurred, with a trend toward less invasive and less extensive dissections. After NSABP B-32, sentinel lymph node biopsy (SLNB) alone became the standard of care for nodal staging of clinically node-negative (cNO) patients. ${ }^{1}$ Then, ACOSOG Z0011 illustrated the safety of SLNB alone in conjunction with adjuvant whole-breast radiation for women with $\mathrm{T} 1$ or $\mathrm{T} 2$ tumors undergoing lumpectomy with two or fewer positive sentinel lymph nodes (SLN), thereby avoiding axillary lymph node dissection (ALND) for $84 \%$ of SLNB-positive patients. ${ }^{2}$ However, there continues to be a marked interest in identifying even less invasive, yet oncologically safe, strategies to establish nodal status.

The use of axillary ultrasound (AUS) was initially described in $1989^{3}$ and its use has expanded significantly during the last 25 years. The potential benefit of AUS is the ability to triage patients with nodal metastases for upfront ALND, thus avoiding the time and cost of a staged SLNB/ ALND. However, this strategy potentially results in unnecessary ALND for women who would otherwise meet the Z0011 criteria. The role of AUS staging is especially

(C) Society of Surgical Oncology 2017

First Received: 20 December 2016; Published Online: 17 January 2017

J. A. Margenthaler, MD, FACS

e-mail: margenthalerj@wudosis.wustl.edu controversial in the setting of patients undergoing neoadjuvant chemotherapy (NAC).

In this study by Barrio et al., ${ }^{4}$ the ability of pre-NAC AUS to predict nodal metastases after NAC was investigated with $402 \mathrm{cN} 0$ patients receiving NAC between 2008 and 2016. Clinical nodal staging was performed by physical examination and collected by chart review. Of the 162 AUS procedures performed, $131(81 \%)$ showed abnormal lymph nodes. Pathologic staging of these lymph nodes was performed via SLNB before NAC, SLNB alone, SLNB then ALND, or ALND alone. The incidence of positive lymph nodes after NAC was higher, yet not significantly different statistically $(p=0.1)$, for patients with an abnormal pre-NAC AUS. However, if abnormal axillary lymph nodes were identified on magnetic resonance imaging (MRI) or positron emission tomography (PET) and computed tomography (CT) before NAC, the patients had a significantly greater chance of having histologically positive lymph nodes $(\mathrm{pN} 1)$ after NAC $(p<0.001$ for both). Differences in tumor biology were found between the patients with pN1 after NAC and the pathologically node-negative ( $\mathrm{pN} 0$ ) patients. Nodal disease was more likely to be identified after NAC in the patients with nonductal histology [odds ratio (OR) 2.93; $p=0.003$ ) and in those with estrogen receptor (ER) positivity (OR 3.94; $p<0.001$ ).

The lower rate of response to NAC among patients with invasive lobular cancer and ER + disease has been illustrated in previous studies. ${ }^{5,6}$ In the entire patient population of the current study, $20 \%$ of the patients with normal axillary lymph nodes identified on pre-NAC imaging were pN1 at the time of definitive surgery. Among 208 patients with abnormal lymph nodes identified by any imaging strategy, $65 \%$ were $\mathrm{pN} 0$ after NAC. The authors concluded that pre-NAC AUS did not predict the need for axillary 
surgery in cNO patients who have already been selected to undergo NAC.

However, we caution against widespread abandonment of AUS in the NAC patient population for several reasons. Current NCCN guidelines suggest that AUS be considered for $\mathrm{cN} 0$ patients before initiation of NAC. ${ }^{7}$ Why does this recommendation exist? Randomized clinical trials investigating the implementation of SLNB after NAC for patients downstaged to $\mathrm{cN} 0$ from clinical node positivity $(\mathrm{cN} 1)$ before NAC initiation provide the framework. For instance, in ACOSOG Z1071, despite the use of dual tracer mapping and excision of at least two SLNs, the false-negative rate (FNR) for SLNB after NAC for previously $\mathrm{cN} 1$ patients remained unacceptably high $(>10 \%)$ unless the axillary lymph node sampled before NAC was resected. ${ }^{8}$ If the clipped node was excised, the FNR dropped to $6.8 \%$. These data suggest that AUS should be considered for patients undergoing NAC to improve the accuracy of the subsequent SLNB and to maintain an acceptable FNR.

The generalizability of the Barrio et al. ${ }^{4}$ data to widespread clinical practice is also of concern based on the demographics and tumor characteristics of its patient population. The median tumor size was $4 \mathrm{~cm}$ (range $1.1-10 \mathrm{~cm})$, and 118 patients $(29 \%)$ had T3 or T4 tumors. No information on the type of breast surgery (mastectomy vs lumpectomy) performed is given. Although the patients in this cohort could not be treated according to ACOSOG Z0011 guidelines due to the delivery of NAC, it is worth commenting that patients with $\mathrm{T} 3$ or T4 tumors and those undergoing mastectomy would also be excluded from ALND deferral because as they also do not meet the Z0011 criteria. In addition, of the 162 patients who did undergo AUS, $81 \%$ had a suspicious AUS, suggesting that this represents a predominantly locally advanced population of patients. Therefore, caution should be taken by the reader to ensure that these results, as well as those of the other studies used in support, are applied only to a matching subset of patients.

The management of the axilla in patients receiving NAC continues to unfold. The final results of ACOSOG Z1071, SENTINA, and SN FNAC ${ }^{9,10}$ will shed light on the most accurate axillary staging methods for $\mathrm{cN} 1$ patients downstaged to cNO after NAC and also will supply long-term data on locoregional failure and overall survival in this patient population.

For the $\mathrm{cN} 0$ patient, it is paramount to identify the goals for the imaging obtained and not create blinded protocols for a pretreatment workup. If tumor biology or tumor size indicate that NAC is warranted, according to current NCCN guidelines as well as what we have learned from ACOSOG Z1071, determination of nodal status with pretreatment AUS, biopsy, and clip placement provides a mechanism to achieve an acceptable SLNB FNR lower than $10 \%$. If no abnormal lymph nodes are identified on pre-NAC imaging, proceeding with an SLNB after NAC is both a feasible and accurate nodal staging technique, as illustrated in NSABP B-27. ${ }^{11}$ For cN0 patients ultimately found to have axillary disease by AUS and biopsy, this knowledge not only may alter the treatment plan toward NAC but also may prompt a staging workup.

Given the low sensitivity (35.5\%) of clinical examination for detecting nodal metastases, ${ }^{12}$ targeted imaging is the only reliable way to assess the axilla before NAC. Although $20 \%$ of the patients in this study with normal lymph nodes before NAC ultimately had nodal metastases, this is consistent with the described FNR (up to $25 \%$ ) of AUS in the literature. ${ }^{13}$ This FNR should not have changed the treatment strategies for the patients in this study. Current data from the same institution suggests a 21 to $97 \%$ pathologic complete response (pCR) rate after NAC depending on tumor biology. ${ }^{14}$ Therefore, even if the lymph node had appeared abnormal initially on pre-NAC ultrasound, one goal of NAC would have been to downstage the axilla and avoid an upfront ALND while identifying the abnormal node for eventual removal at the time of post-NAC SLNB.

The sonographic appearance of a lymph node cannot dictate response to NAC. As described by the authors of this report, only tumor biology has been shown to predict likelihood of pCR. The AUS procedure should be used to guide treatment strategies by assisting with the establishment of accurate clinical staging as an adjunct to clinical examination. Due to its subjective interpretation and the variety of ways that a lymph node metastasis can present sonographically, further efforts to develop algorithms for predicting nodal disease, such as that described by Qiu et al., ${ }^{15}$ should be encouraged. In addition, the results of the ongoing SOUND trial, which is randomizing cN0 patients with a normal AUS to SLNB versus observation, also will offer more information on the clinical utility of AUS. ${ }^{16}$

Therefore, we argue that AUS should not yet be removed from the pre-NAC imaging armamentarium. Establishing the clinical nodal status with physical examination alone is fraught with missed opportunities to identify patients with nodal disease that would otherwise alter the course of their treatment. The use of NAC has significantly shifted from a predominantly locally advanced, inoperable population to patients with earlier, operable disease. In the era of targeted therapy, many $\mathrm{T} 1 /$ $\mathrm{T} 2$ patients become eligible for NAC based on the nodal status. We should strive to identify the patient characteristics, tumor biology, and cancer subtypes that mark patients who would benefit from selective AUS. Until such discriminatory markers are identified, we will continue using AUS routinely to determine the most accurate 
clinical stage, with the goal of minimizing treatment morbidity and improving breast cancer-related outcomes.

\section{REFERENCES}

1. Krag D, Anderson SJ, Julian TB, Brown AM, Harlow SP, Costantino JP, et al. Sentinel lymph node resection compared with conventional axillary lymph node dissection in clinically node-negative patients with breast cancer: overall survival findings from the NSABP B-32 randomised phase 3 trial. Lancet Oncol. 2010;11:927-33.

2. Giuliano AE, Hunt KK, Ballman KV, Beitsch PD, Whitworth PW, Blumencranz PW, et al. Axillary dissection vs no axillary dissection in women with invasive breast cancer and sentinel node metastasis: a randomized clinical trial. JAMA. 2011;305:569-75.

3. Tate JJ, Lewis V, Archer T, Guyer PG, Royle GT, Taylor I. Ultrasound detection of axillary lymph node metastases in breast cancer. Eur J Surg Oncol. 1989;15:139-41.

4. Barrio AV, Mamtani A, Eaton A, et al. Is routine axillary imaging necessary in clinically node-negative patients undergoing neoadjuvant chemotherapy? Ann Surg Oncol. 2017. doi:10.1245/ s10434-017-5765-y.

5. Cristofanilli M, Gonzalez-Angulo A, Sneige N, Kau SW, Broglio $\mathrm{K}$, Theriault RL, et al. Invasive lobular carcinoma classic type: response to primary chemotherapy and survival outcomes. J Clin Oncol. 2005;23:41-8.

6. Lips EH, Mulder L, deRonde JJ, Mandjes IA, Vincent A, Vrancken Peeters MT, et al. Neoadjuvant chemotherapy in ER + HER2 - breast cancer: response prediction based on immunohistochemical and molecular characteristics. Breast Cancer Res Treat. 2012;131:827-36.

7. Gradishar WJ, Anderson BO, Balassanian R, Blair SL, Burstein HJ, Cyr A, et al. Breast cancer version 2.2016. https://www.nccn. org/professionals/physician_gls/pdf/breast.pdf. Accessed 8 Dec 2016.

8. Boughey JC, Ballman KV, Le-Petross HT, McCall LM, Mittendorf EA, Ahrendt GM, et al. Identification and resection of clipped node decreases the false-negative rate in patients presenting with node-positive breast cancer (T0-T4, N1-N2) who receive neoadjuvant chemotherapy. Ann Surg. 2016;263:802-7.

9. Kuehn T, Bauerfeind I, Fehm T, Fleige B, Hausschild M, Helms $\mathrm{G}$, et al. Sentinel lymph node biopsy in patients with breast cancer before and after neoadjuvant chemotherapy (SENTINA): a prospective, multicenter cohort study. Lancet Oncol. 2013;14:609-18.

10. Boileau JF, Poirier B, Basik M, Holloway M, Gaboury I, Sideris L, et al. Sentinel node biopsy after neoadjuvant chemotherapy in biopsy-proven node-positive breast cancer: the SN FNAC study. J Clin Oncol. 2014;33:258-64.

11. Mamounas EP, Brown A, Anderson S, Smith R, Julian T, Miller $B$, et al. Sentinel lymph node biopsy after neoadjuvant chemotherapy in breast cancer: results from National Surgical Breast and Bowel Project Protocol B-27. J Clin Oncol. 2005;23:2694-702.

12. Valente SA, Levine GM, Silverstein MJ, Rayhanabad JA, WengGrumley JC, Ji L, et al. Accuracy of predicting axillary lymph node positivity by physical examination, mammography, ultrasonography, and magnetic resonance imaging. Ann Surg Oncol. 2012;19:1825-30.

13. Diepstraten SC, Sever AR, Buckens CF, Veldhuis WB, vanDalen $\mathrm{T}$, van den Bosch MA, et al. Value of preoperative ultrasoundguided axillary lymph node biopsy for preventing completion axillary lymph node dissection in breast cancer: a systematic review and meta-analysis. Ann Surg Oncol. 2014;21:51-9.

14. Mamtani A, Barrio AV, King TA, Van Zee KJ, Plitas G, Pilewskie M, et al. How often does neoadjuvant chemotherapy avoid axillary dissection in patients with histologically confirmed nodal metastases? Results of a prospective study. Ann Surg Oncol. 2016;23:3467-74.

15. Qiu SQ, Zeng HC, Zhang F, Chen C, Huang WH, Pleijhuis RG, et al. A nomogram to predict the probability of axillary lymph node metastasis in early breast cancer patients with positive axillary ultrasound. Sci Rep. 2016;6:21196.

16. Gentilini O1, Veronesi U. Abandoning sentinel lymph node biopsy in early breast cancer? A new trial in progress at the European Institute of Oncology of Milan (SOUND: sentinel node vs observation after axillary UltraSouND). Breast. 2012;21:678-81. 